\title{
Preliminary Deep Water Results in Single-Beacon One-Way-Travel-Time Acoustic Navigation for Underwater Vehicles
}

\author{
Sarah E. Webster*, Ryan M. Eustice ${ }^{\dagger}$, Hanumant Singh ${ }^{\ddagger}$, Louis L. Whitcomb* \\ *Department of Mechanical Engineering \\ Johns Hopkins University, Baltimore, MD 21218 \\ email: \{swebster,llw\}@jhu.edu \\ ${ }^{\dagger}$ Department of Naval Architecture and Marine Engineering \\ University of Michigan, Ann Arbor, MI, 48109 \\ email: eustice@umich.edu \\ $\ddagger$ Department of Applied Ocean Physics \& Engineering \\ Woods Hole Oceanographic Institution, Woods Hole, MA 02543 \\ email: hanu@whoi.edu
}

\begin{abstract}
This paper reports the development and experimental evaluation of a novel navigation system for underwater vehicles that employs Doppler sonar, synchronous clocks, and acoustic modems to achieve simultaneous acoustic communication and navigation. The system reported herein, which is employed to renavigate the vehicle in post-processing, forms the basis for a vehicle-based real-time navigation system. Existing high-precision absolute navigation techniques for underwater vehicles are impractical over long length scales and lack scalability for simultaneously navigating multiple vehicles. The navigation method reported in this paper relies on a single moving reference beacon, eliminating the requirement for the underwater vehicle to remain in a bounded navigable area. The use of underwater modems and synchronous clocks enables range measurements based on one-way time-of-flight information from acoustic data packet broadcasts. The acoustic data packets are broadcast from the single, moving reference beacon and can be received simultaneously by multiple vehicles within acoustic range. We report experimental results from the first deep-water evaluation of this method using data collected from an autonomous underwater vehicle (AUV) survey carried out in $4000 \mathrm{~m}$ of water on the southern Mid-Atlantic Ridge. We report a comparative experimental evaluation of the navigation fixes provided by the proposed synchronous acoustic navigation system in comparison to navigation fixes obtained by an independent conventional long baseline acoustic navigation system.
\end{abstract}

\section{INTRODUCTION}

This paper reports the development and experimental evaluation of a novel navigation system for underwater vehicles that employs Doppler sonar, synchronous clocks, and acoustic modems to achieve simultaneous acoustic communication and navigation. Our goal is to enable highprecision absolute navigation of underwater vehicles over length scales of $\mathcal{O}(1-100 \mathrm{~km})$ without requiring fixed navigation reference beacons. Available strap-down sensors such as Doppler velocity logs (DVLs) and inertial measurement units (IMUs) measure vehicle velocities and accelerations in pose and attitude, which can be integrated to estimate relative change in vehicle position. Unaided IMU and DVL navigation methods estimate local displacement with errors that are unbounded over time, thus they require additional auxiliary navigation methods to provide error correction and an absolute georeference.

Bounded-error navigation is currently achieved with the aid of systems such as long baseline navigation (LBL) that require external, fixed reference beacons, additional survey time, and have a range of only $5-10 \mathrm{~km}$. In addition, some LBL systems suffer a lack of scalability because only one vehicle can interrogate the network of acoustic beacons at a time. This effectively decreases the rate at which a given vehicle can receive navigation fixes to $1 / \mathrm{N}$, where $\mathrm{N}$ is the number of vehicles using the network.

In contrast, synchronous-clock one-way-travel-time (OWTT) acoustic navigation is a navigation technique that relies on ranges estimated from time-of-flight information of acoustic data packets between a reference beacon with a known, though not necessarily stationary, location and the vehicle [13] [14]. This method provides both bounded-error position estimates and, with a moving reference beacon, long range $\mathcal{O}(100 \mathrm{~km})$ capabilities. OWTT navigation provides scalability as well, allowing all vehicles within acoustic range to simultaneously use the same acoustic data packet broadcast independent of the number of vehicles within acoustic range. The implementation of the method described herein requires the use of underwater acoustic modems on both the reference beacon (in our case the ship) and the vehicle, as well as a precision clock to synchronize the modems. The state estimator reported in this paper forms the basis for a real-time, vehicle-based state estimator. The implementation reported here is for post-processing vehicle position; the modifications necessary for real-time navigation are noted in the text. An alternate method of navigation using synchronous-pingers and one-way-travel-times to calculate range-rate is discussed in [44].

The remainder of this paper is organized as follows: Section II describes previous work in the area of singlebeacon navigation based on range measurements. Section III reports an overview of synchronous-clock one-way-traveltime navigation. Section IV briefly reviews the extended Kalman filter and reports the mathematical framework for 
the extended Kalman filter formulated for the work presented herein. Section V reports field results from the deep-water sea trials, and Section VI contains concluding remarks.

\section{Previous Work}

The majority of prior literature in this area report proposed estimation algorithms and the results of numerical simulations of these algorithms. Only a few report experimental evaluations of the proposed algorithms, and even fewer employ independent navigation methods to evaluate quantitatively the accuracy of the proposed methods.

The earliest formulation of vehicle navigation using ranges from a single beacon that is known to the authors is reported in [43]. This approach employs least-squares to solve for the vehicle's unknown initial position and a constant-velocity unknown current; additionally, a linear-algebra based observability analysis is also reported.

Range-only localization methods used for estimating the position of a target are addressed by [41] and [46]. In [41] the authors compute the theoretical Cramér-Rao bound and compare it to the performance of a maximum-likelihood estimator (MLE), an extended Kalman filter(EKF), and a regularized particle filter during field tests. In [46] the author addresses the observability of the target-tracker problem using the Fisher information matrix and reports simulation results using an EKF. In related work [1] implements the EKF from [46] and reports simulation results.

The use of EKFs for homing and single-beacon navigation, initialized by least-squares, is reported in [3], [4], [48] for both simulation and field trials. In [4] the authors also report a simulated two-vehicle system using a cascaded approach in which the second vehicle navigates relative to the first vehicle using inter-vehicle range measurements.

The papers [32]-[34] report an error state EKF for singlebeacon navigation based on error models of the vehicle's inertial navigation system. The authors report results using a combination of field and simulation data.

More recent least-squares solutions are reported in [23] and [31], the former using ad hoc iterative techniques to estimate course, the later reporting a method for advancing multiple single-beacon fixes along the vehicle's estimated trackline to simulate a multi-beacon fix.

An extended set-valued observer is reported in [37]. The authors show this observer to provide bounds on the estimation error in the presence of non-linearities in the model and non-Gaussian noise, guaranteeing that the true vehicle position is contained within the estimator's predicted error covariance ellipsoid when linearization error and noise are correctly characterized.

Several different methods for addressing the observability of single-beacon range-only navigation are reported in the literature. The papers [16]-[19] report an observability analysis employing limiting systems to assess uniform observability, and derive sufficient conditions for the existence of an observer with exponentially decaying estimation error for the cases of both known and unknown ambient currents. The authors report field results from their implementation of an EKF. In related work [36] extends the EKF reported in [16]-[19] to three-dimensional coordinates with simulation results.

A concise observability analysis in continuous time using Lie derivatives to compute conditions for which the system has local weak observability is reported in [42]. In [25] the authors report an algebraic analysis showing local uniform observability based on signal estimation techniques, though the lack of an estimation model disallows the computation of an updated position in the absence of a new measurement.

The papers [39], [40] address range-only navigation of underwater vehicles in a simultaneous localization and mapping (SLAM) framework and report experimental results. In this formulation multiple beacons are used but a priori beacon location is not known. The papers [5], [6] address cooperative localization of multiple underwater and surface vehicles in a SLAM framework.

Multi-beacon, range-only navigation for terrestrial vehicles in a SLAM framework is addressed in [11], [12], [27][30] using radio-frequency beacons for range measurement, in [38] using audible sound, in [35], [47] using wireless sensor networks, and in [8], [9] with an unspecified range sensor.

The work reported in this paper extends the results reported in [13], [14], which report the theory and first experimental results in synchronous-clock one-way-traveltime acoustic navigation with an acoustic modem and precision timing board. These two papers employ a maximumlikelihood estimator and report field results from shallowwater sea trials.

\section{Synchronous-Clock One-Way-Travel-Time ACOUSTIC NAVIGATION}

This section reports an overview of synchronous-clock one-way-travel-time acoustic navigation followed by the details of our implementation during sea trials.

\section{A. Methodology}

Synchronous-clock one-way-travel-time acoustic navigation relies on range estimation from the time-of-flight (TOF) of acoustic data packets propagating between a vehicle and one or more reference beacons at known locations in order to provide a reference to the world frame. Between range measurements, vehicle position is estimated using velocity, acceleration, and attitude measurements.

Figure 1 depicts a ship-based acoustic modem broadcasting acoustic data packets to multiple underwater vehicles. The acoustic data packets are transmitted from a known, but not necessarily stationary, location. Using an underwater acoustic modem we encode the sender's geodetic location and the time-of-launch (TOL) in the acoustic data packet. The time-of-arrival (TOA) of this acoustic data packet at the receiver, combined with the decoded TOL and position information in the acoustic data packet, are used to estimate range.

Because we calculate TOF from the difference between the sender's time and the receiver's time, it is crucial that the clocks on the sender and receiver be synchronized throughout 


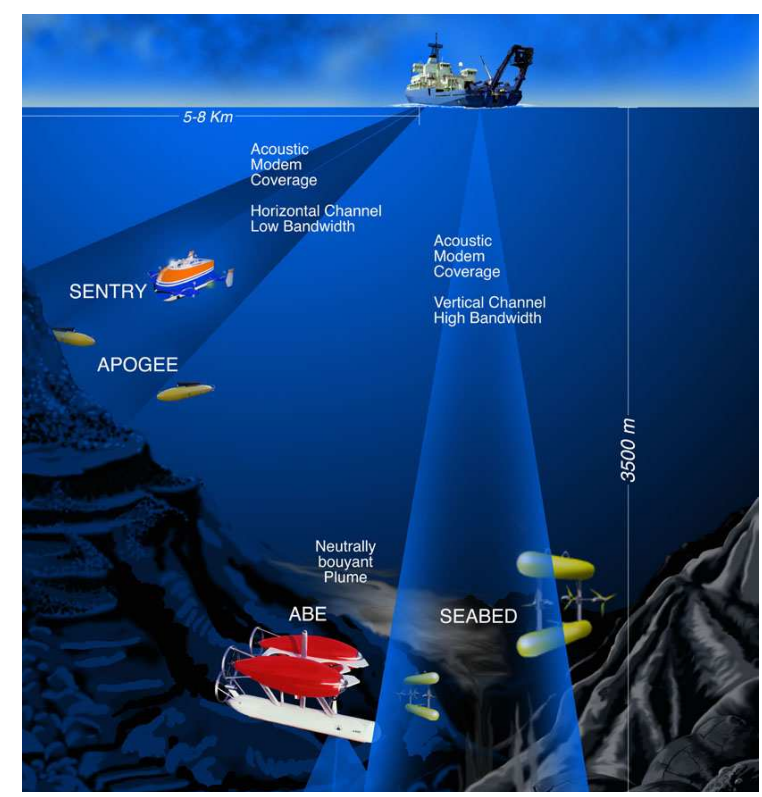

Fig. 1. Acoustic data packet broadcast from the ship to multiple vehicles.

the dive to within an acceptable tolerance. The time keeping problem and our solution is discussed in more detail in Section III-B.

\section{B. OWTT Navigation Implementation}

For sea trials, a ship equipped with a global positioning system (GPS) receiver served as a moving reference beacon. Both the ship and the vehicle were equipped with micromodems designed and built at Woods Hole Oceanographic Institution (WHOI) for sending and receiving acoustic data packets through the water column [15]. The Micro-modem supports 32-byte long binary data acoustic packets, into which we encode the sender's location and the TOL of the acoustic data packet [15]. Using the modem's synchronous navigation mode, the modem is configured to transmit acoustic data packets beginning at the top of the second, so that only the name of the top-of-the-second is required to know the TOL.

Given that all acoustic messages are transmitted at the top of the second, an additional source of range information is also available when using the WHOI Micro-modem. The Micro-modem can be configured so that every acoustic message received, not just acoustic data packets with specific OWTT navigation information, generates a TOA message. Provided that the range from the vehicle to the ship is known approximately (i.e. to within $\sim 1500 \mathrm{~m}$ ) the expected integer number of seconds in the TOF measurement is known. The integer seconds can be combined with the fractional seconds from the TOA message to provide a TOF measurement without precise knowledge of the sender's location.

In post-processing, the complete trajectory of the ship is available, allowing for range measurements from the ship's true location as reported by the GPS. In real time, when range measurements are calculated at the vehicle, the ship's position would be estimated using only the ship's position information that has been transmitted acoustically to the vehicle.

For a precision timing board, the vehicle used a PPSBoard, originally reported in [13], [14]. The PPSBoard uses a low-power, temperature-compensated precision clock from SeaScan Inc. to provide precise time keeping. The SeaScan clock has a maximum drift rate of approximately $1 \mathrm{~ms}$ over $14 \mathrm{hrs}$. A $1 \mathrm{~ms}$ timing error results in a $1.5 \mathrm{~m}$ error in range, which is acceptable for most error tolerances. Prior to each vehicle dive, the PPSBoard is synchronized to coordinated universal time (UTC) via GPS. Further details on the PPSBoard and precision clocks are addressed in [13], [14].

\section{EXTENDED KALMAN Filter}

This section briefly reviews the formulation of the extended Kalman filter (EKF) employed in this paper followed by the details of our implementation.

\section{A. Review of EKF Formulation}

The extended Kalman filter is a non-optimal filter that applies the general approach of the Kalman filter, [26], to nonlinear plants by linearizing the plant process and observation models along the trajectory of the system. The formulation reported here is for a nonlinear plant with discrete observations [20]. Consider the nonlinear plant process and observation model

$$
\begin{aligned}
\dot{\boldsymbol{x}}(t) & =\boldsymbol{f}(\boldsymbol{x}(t), t)+\boldsymbol{w}(t) \\
\boldsymbol{z}_{k} & =\boldsymbol{h}_{k}\left(\boldsymbol{x}\left(t_{k}\right)\right)+\boldsymbol{v}_{k}, k=1,2, \cdots
\end{aligned}
$$

where $\boldsymbol{x}(t)$ is the state, $\boldsymbol{z}_{k}$ is the measurement at time step $t_{k}$, and $\boldsymbol{w}(t) \sim \mathcal{N}(0, \boldsymbol{Q}(t))$ and $\boldsymbol{v}_{k} \sim \mathcal{N}\left(0, \boldsymbol{R}_{k}\right)$ are independent zero-mean Gaussian noise.

The EKF framework used herein employs a discretetime linearization of the process model, resulting in process prediction equations

$$
\begin{aligned}
\hat{\boldsymbol{x}}_{k \mid k-1} & =\boldsymbol{F}_{k} \hat{\boldsymbol{x}}_{k-1 \mid k-1}+\boldsymbol{B}_{k} \boldsymbol{u}_{k-1} \\
\boldsymbol{P}_{k \mid k-1} & =\boldsymbol{F}_{k} \boldsymbol{P}_{k-1 \mid k-1} \boldsymbol{F}_{k}^{\top}+\boldsymbol{Q}_{k}
\end{aligned}
$$

where $\boldsymbol{F}_{k}$ is the discrete-time linear state transition matrix, $\boldsymbol{B}_{k}$ is the discrete-time linear input matrix, $\boldsymbol{Q}_{k}$ is the discrete-time error covariance, and $\boldsymbol{u}_{k-1}$ is the piecewiseconstant input at time step $t_{k-1}$.

The measurement update equations for the extended Kalman filter are

$$
\begin{aligned}
\hat{\boldsymbol{x}}_{k \mid k} & =\hat{\boldsymbol{x}}_{k \mid k-1}+\boldsymbol{K}_{k}\left(\boldsymbol{z}_{k}-\boldsymbol{h}_{k}\left(\hat{\boldsymbol{x}}_{k \mid k-1}\right)\right) \\
\boldsymbol{P}_{k \mid k} & =\boldsymbol{P}_{k \mid k-1}-\boldsymbol{K}_{k} \boldsymbol{H}_{k} \boldsymbol{P}_{k \mid k-1}
\end{aligned}
$$

where $\boldsymbol{H}_{k}$ is the Jacobian of $\boldsymbol{h}_{k}$ at time step $t_{k}$

$$
\boldsymbol{H}_{k}=\left.\frac{\partial \boldsymbol{h}_{k}\left(\boldsymbol{x}\left(t_{k}\right)\right)}{\partial \boldsymbol{x}\left(t_{k}\right)}\right|_{\boldsymbol{x}\left(t_{k}\right)=\hat{\boldsymbol{x}}_{k \mid k-1}}
$$

and $\boldsymbol{K}_{k}$ is the Kalman gain at time step $t_{k}$, given by

$$
\boldsymbol{K}_{k}=\boldsymbol{P}_{k \mid k-1} \boldsymbol{H}_{k}^{\top}\left(\boldsymbol{H}_{k} \boldsymbol{P}_{k \mid k-1} \boldsymbol{H}_{k}^{\top}+\boldsymbol{R}_{k}\right)^{-1} .
$$




\section{B. EKF Implementation}

We employ an extended Kalman filter to fuse depth, gyrocompass, and Doppler velocity measurements from the vehicle, position and attitude measurements from the ship, and range measurements between the vehicle and the ship.

The EKF described herein is designed for post-processing of previous dive data. The current and previous states of both the ship and the vehicle are available in postprocessing, and are processed simultaneously, which enables range measurements from both the ship to the vehicle and the vehicle to the ship to be used in the EKF. In a realtime implementation, range measurements made at the ship, i.e. range measurements resulting from acoustic messages sent from the vehicle to the ship, would not be available to a vehicle-based state estimator. In addition, a real-time vehicle-based implementation would only have knowledge of anachronistic, sub-sampled measurements from the ship that were transmitted acoustically, resulting in an $\mathcal{O}(1-5 \mathrm{sec})$ delay and a relative paucity of ship-based measurements available at the vehicle.

We use a depth-weighted average sound velocity when calculating range from time-of-flight (TOF) information. We assume that this speed of sound is constant over the range of depths the vehicle traverses while near the sea floor carrying out the survey.

\section{State Description}

The state vector consists of the current vehicle estimate, $\boldsymbol{x}_{v}$, the current ship estimate, $\boldsymbol{x}_{s}$, and a fixed-length queue of delayed states, recorded at the top of the second, for the most recent $n$ seconds, denoted $\boldsymbol{x}_{v-i}$ for $i \in[1, . ., n]$.

$$
\boldsymbol{x}=\left[\boldsymbol{x}_{v}, \boldsymbol{x}_{s}, \boldsymbol{x}_{v-1}, \boldsymbol{x}_{s-1}, \cdots, \boldsymbol{x}_{v-n}, \boldsymbol{x}_{s-n}\right]^{\top}
$$

In practice $n=6$ for our implementation assuming a maximum slant range of $6000 \mathrm{~m}$ between the vehicle and the ship.

The current ship state contains xy-position, heading, and the respective velocities.

$$
\boldsymbol{x}_{s}=\left[x_{s}, y_{s}, \theta_{s}, \dot{x}_{s}, \dot{y}_{s}, \dot{\theta}_{s}\right]^{\top}
$$

The current vehicle state contains local-level pose and attitude, as well as body-frame linear and angular velocities

$$
\begin{gathered}
\boldsymbol{x}_{v}=\left[\boldsymbol{s}^{\top}, \boldsymbol{\varphi}^{\top}, \boldsymbol{v}^{\top}, \boldsymbol{\omega}^{\top}\right]^{\top} \\
\boldsymbol{s}=\left[\begin{array}{c}
x \\
y \\
z
\end{array}\right] \boldsymbol{\varphi}=\left[\begin{array}{c}
\phi \\
\theta \\
\psi
\end{array}\right] \boldsymbol{v}=\left[\begin{array}{c}
u \\
v \\
w
\end{array}\right] \boldsymbol{\omega}=\left[\begin{array}{l}
p \\
q \\
r
\end{array}\right]
\end{gathered}
$$

where $s$ is the local-level vehicle pose in the local frame, $\varphi$ is the local-level vehicle attitude (Euler roll, pitch, heading), $\boldsymbol{v}$ is the body-frame linear velocity, and $\boldsymbol{\omega}$ is the body-frame angular velocity.

The delayed states contain only a subset of the elements in the current state vector-the vehicle's xyz-position and ship's-xy position and heading from a previous state.

$$
\begin{aligned}
& \boldsymbol{x}_{v-i}=[x(t-i), y(t-i), z(t-i)]^{\top} \\
& \boldsymbol{x}_{s-i}=\left[x_{s}(t-i), y_{s}(t-i), \theta_{s}(t-i)\right]^{\top}
\end{aligned}
$$

Delayed states are necessary for causal processing because of the time required for an acoustic data packet to propagate from the sender to the receiver. The TOF measured is thus between a previous state of the sender and the current state of the receiver. The modems are configured to initiate acoustic data packets only at the top-of-the-second, so it is only at the top-of-the-second that we record delayed states.

\section{Process Model}

We assume a constant velocity process model for both the vehicle and the ship

$$
\begin{aligned}
& \dot{\boldsymbol{x}}_{v}=\underbrace{\left[\begin{array}{cccc}
\mathbf{0} & \mathbf{0} & \boldsymbol{R}(\boldsymbol{\varphi}) & \mathbf{0} \\
\mathbf{0} & \mathbf{0} & \mathbf{0} & \mathcal{J}(\varphi) \\
\mathbf{0} & \mathbf{0} & \mathbf{0} & \mathbf{0} \\
\mathbf{0} & \mathbf{0} & \mathbf{0} & \mathbf{0}
\end{array}\right]}_{\boldsymbol{f}\left(\boldsymbol{x}_{v}(t)\right)} \boldsymbol{x}_{v}+\underbrace{\left[\begin{array}{cc}
\mathbf{0} & \mathbf{0} \\
\mathbf{0} & \mathbf{0} \\
\boldsymbol{I} & \mathbf{0} \\
\mathbf{0} & \boldsymbol{I}
\end{array}\right]}_{\boldsymbol{G}_{v}} \boldsymbol{w}_{v} \\
& \dot{\boldsymbol{x}}_{s}=\underbrace{\left[\begin{array}{ll}
\mathbf{0} & \boldsymbol{I} \\
\mathbf{0} & \mathbf{0}
\end{array}\right]}_{\boldsymbol{F}_{s}} \boldsymbol{x}_{s}+\underbrace{\left[\begin{array}{c}
\mathbf{0} \\
\boldsymbol{I}
\end{array}\right]}_{\boldsymbol{G}_{s}} \boldsymbol{w}_{s}
\end{aligned}
$$

where $\boldsymbol{R}(\varphi)$ is the transformation between local-level and body-frame linear velocities, $\mathcal{J}(\varphi)$ is the transformation between body-frame angular velocities and Euler rates, and $\boldsymbol{w}_{v} \sim \mathcal{N}\left(0, \boldsymbol{Q}_{v}\right)$ and $\boldsymbol{w}_{s} \sim \mathcal{N}\left(0, \boldsymbol{Q}_{s}\right)$ are independent zeromean Gaussian process noise in the respective acceleration terms. $\boldsymbol{R}(\varphi)$ and $\mathcal{J}(\varphi)$ are found by solving

$$
\begin{aligned}
\boldsymbol{R}(\boldsymbol{\varphi})= & \boldsymbol{R}_{\psi}^{\top} \boldsymbol{R}_{\theta}^{\top} \boldsymbol{R}_{\phi}^{\top} \\
\boldsymbol{R}_{\psi}= & {\left[\begin{array}{ccc}
\cos \psi & \sin \psi & 0 \\
-\sin \psi & \cos \psi & 0 \\
0 & 0 & 1
\end{array}\right] } \\
\boldsymbol{R}_{\theta}= & {\left[\begin{array}{ccc}
\cos \theta & 0 & -\sin \theta \\
0 & 1 & 0 \\
\sin \theta & 0 & \cos \theta
\end{array}\right] } \\
\boldsymbol{R}_{\phi}= & {\left[\begin{array}{ccc}
1 & 0 & 0 \\
0 & \cos \phi & \sin \phi \\
0 & -\sin \phi & \cos \phi
\end{array}\right] }
\end{aligned}
$$

and

$$
\begin{aligned}
& \boldsymbol{\omega}=\left[\begin{array}{c}
\dot{\phi} \\
0 \\
0
\end{array}\right]+R_{\phi}\left[\begin{array}{c}
0 \\
\dot{\theta} \\
0
\end{array}\right]+R_{\phi} R_{\theta}\left[\begin{array}{c}
0 \\
0 \\
\dot{\psi}
\end{array}\right] \\
& =\underbrace{\left[\begin{array}{ccc}
1 & 0 & -\sin \theta \\
0 & \cos \phi & \sin \phi \cos \theta \\
0 & -\sin \phi & \cos \phi \cos \theta
\end{array}\right]}_{\mathcal{J}^{-1}} \dot{\varphi}
\end{aligned}
$$

\section{E. Process Prediction and Augmentation}

The process model is linearized and discretized according to standard methods [7]. The resulting discrete-time linearized vehicle process model is

$$
\boldsymbol{x}_{v_{k+1}}=\boldsymbol{F}_{v_{k}} \boldsymbol{x}_{v_{k}}+\boldsymbol{B}_{v_{k}} \boldsymbol{u}_{k}+\boldsymbol{w}_{v_{k}}
$$


where

$$
\begin{aligned}
\boldsymbol{F}_{v_{k}} & =e^{\boldsymbol{F}_{x} T} \\
\boldsymbol{B}_{v_{k}} & =\int_{t_{k}}^{t_{k+1}} e^{\boldsymbol{F}_{x}\left(t_{k+1}-\tau\right)} d \tau,
\end{aligned}
$$

$\boldsymbol{F}_{x}$ is the Jacobian with respect to $\boldsymbol{x}_{v}$

$$
\boldsymbol{F}_{x}=\left.\frac{\partial \boldsymbol{f}\left(\boldsymbol{x}_{v}(t)\right)}{\partial \boldsymbol{x}_{v}(t)}\right|_{\boldsymbol{x}_{v}(t)=\boldsymbol{x}_{v}\left(t_{k}\right)},
$$

$\boldsymbol{u}_{k}$ is a constant resulting from the linearization that can be treated as a piecewise-constant input

$$
\boldsymbol{u}_{k}=f\left(\boldsymbol{x}_{v_{k}}\right)-\boldsymbol{F}_{x} \boldsymbol{x}_{v_{k}},
$$

and $T$ is the sampling period. The discretized vehicle process noise, $\boldsymbol{w}_{v_{k}}$, is zero-mean Gaussian,

$$
\begin{gathered}
\boldsymbol{w}_{v_{k}} \sim \mathcal{N}\left(0, \boldsymbol{Q}_{v_{k}}\right) \\
\boldsymbol{Q}_{v_{k}}=\int_{0}^{T} e^{\boldsymbol{F}_{x}(T-\tau)} \boldsymbol{G} \boldsymbol{Q}_{v} \boldsymbol{G}^{\top} e^{\boldsymbol{F}_{x}^{\top}(T-\tau)} d \tau
\end{gathered}
$$

The discrete-time linearized process model for the ship is

$$
\begin{aligned}
\boldsymbol{x}_{s_{k+1}} & =\boldsymbol{F}_{s_{k}} \boldsymbol{x}_{s_{k}}+\boldsymbol{w}_{s_{k}} \\
\boldsymbol{F}_{s_{k}} & =e^{\boldsymbol{F}_{\boldsymbol{s}} T}=\left[\begin{array}{cc}
\boldsymbol{I} & \boldsymbol{I} T \\
\mathbf{0} & \boldsymbol{I}
\end{array}\right]
\end{aligned}
$$

where $\boldsymbol{F}_{s}$ is defined in (16). The discretized process noise for the ship, $\boldsymbol{w}_{s_{k}}$, is zero-mean Gaussian,

$$
\boldsymbol{w}_{s_{k}} \sim \mathcal{N}\left(0, \boldsymbol{Q}_{s_{k}}\right)
$$

where the covariance matrix simplifies to

$$
\boldsymbol{Q}_{s_{k}}=\left[\begin{array}{cc}
\frac{1}{3} T^{3} & \frac{1}{2} T^{2} \\
\frac{1}{2} T^{2} & T
\end{array}\right] \boldsymbol{Q}_{s}
$$

due to the structure of $\boldsymbol{F}_{s_{k}}$.

The complete state process prediction is written in terms of the full state vector of the system defined in (9). Combining the discrete-time linearized vehicle and ship process models (19) and (26), and substituting them into the discretetime linearized Kalman process prediction equation (3), the complete state process prediction becomes

$\hat{\boldsymbol{x}}_{k+1 \mid k}=\left[\begin{array}{ccccc}\boldsymbol{F}_{v_{k}} & 0 & 0 & \cdots & 0 \\ 0 & \boldsymbol{F}_{s_{k}} & 0 & \cdots & 0 \\ 0 & 0 & \boldsymbol{I} & \cdots & 0 \\ \vdots & \vdots & \vdots & \ddots & \vdots \\ 0 & 0 & 0 & \cdots & \boldsymbol{I}\end{array}\right] \hat{\boldsymbol{x}}_{k \mid k}+\left[\begin{array}{c}\boldsymbol{B}_{v_{k}} \boldsymbol{u}_{k} \\ 0 \\ 0 \\ \vdots \\ 0\end{array}\right]$

Note that the delayed states do not change during this process update.

A modified process prediction is necessary at the top of the second when state augmentation is done in concert with the process prediction. During this modified prediction step, in addition to predicting forward the current vehicle state, a copy of the relevant elements of the current state (before the prediction) is added to the state vector while simultaneously marginalizing out the oldest delayed state, i.e. $\left(\boldsymbol{x}_{v-n}, \boldsymbol{x}_{s-n}\right)$

$$
\begin{gathered}
\hat{\boldsymbol{x}}_{k+1 \mid k}= \\
{\left[\begin{array}{ccccccc}
\boldsymbol{F}_{v_{k}} & 0 & 0 & \cdots & 0 & 0 & 0 \\
0 & \boldsymbol{F}_{s_{k}} & 0 & \cdots & 0 & 0 & 0 \\
\boldsymbol{J}_{v} & 0 & 0 & \cdots & 0 & 0 & 0 \\
0 & \boldsymbol{J}_{s} & 0 & \cdots & 0 & 0 & 0 \\
0 & 0 & \boldsymbol{I} & \cdots & 0 & 0 & 0 \\
\vdots & \vdots & \vdots & \ddots & \vdots & \vdots & \vdots \\
0 & 0 & 0 & \cdots & \boldsymbol{I} & 0 & 0
\end{array}\right] \hat{\boldsymbol{x}}_{k \mid k}+\left[\begin{array}{c}
\boldsymbol{B}_{v_{k}} \boldsymbol{u}_{k} \\
0 \\
0 \\
0 \\
0 \\
\vdots \\
0
\end{array}\right]}
\end{gathered}
$$

where $\boldsymbol{J}_{v}$ and $\boldsymbol{J}_{s}$ are defined such that only the relevant elements of the current ship and vehicle states are recorded.

$$
\begin{gathered}
\underbrace{\left[\begin{array}{llll}
\boldsymbol{I} & 0 & 0 & 0
\end{array}\right]}_{\boldsymbol{J}_{v}} \underbrace{\left[\begin{array}{c}
(x, y, z)^{\top} \\
(\phi, \theta, \psi)^{\top} \\
(u, v, w)^{\top} \\
(p, q, r)^{\top}
\end{array}\right]}_{\boldsymbol{x}_{v}}=\left[\begin{array}{l}
x \\
y \\
z
\end{array}\right] \\
\underbrace{\left[\begin{array}{ll}
\boldsymbol{I} & 0
\end{array}\right]}_{\boldsymbol{J}_{s}} \underbrace{\left[\begin{array}{c}
\left(x_{s}, y_{s}, \theta_{s}\right)^{\top} \\
\left(\dot{x}_{s}, \dot{y}_{s}, \dot{\theta}_{s}\right)^{\top}
\end{array}\right]}_{\boldsymbol{x}_{s}}=\left[\begin{array}{c}
x_{s} \\
y_{s} \\
\theta_{s}
\end{array}\right]
\end{gathered}
$$

\section{F. Measurement Model and Update}

The range measurement from the ship's modem to the vehicle's modem is a nonlinear function of current vehicle state and a delayed ship state. For simplicity of notation, we assume here that the modems are located at the origin of their respective local frames and that the ship's modem has a depth of 0 . We define a combined pose vector $\boldsymbol{x}_{p}$ that contains $\boldsymbol{x}_{v_{p}}$, the current vehicle pose (xyz-position), and $\boldsymbol{x}_{s_{p}}$, the delayed ship pose (xy-position, assuming a depth of 0 )

$$
\boldsymbol{x}_{p}=\left[\begin{array}{c}
\boldsymbol{x}_{v_{p}} \\
\boldsymbol{x}_{s_{p}}
\end{array}\right]=\left[\begin{array}{c}
x \\
y \\
z \\
x_{s} \\
y_{s} \\
0
\end{array}\right]
$$

where $\boldsymbol{x}_{v_{p}}$ is a subset of the current vehicle state and $\boldsymbol{x}_{s_{p}}$ is a subset of the delayed ship state.

The measurement equation for the ship to vehicle range in terms of $\boldsymbol{x}_{p}$ is

$$
z_{k}=\sqrt{\left(\boldsymbol{x}_{v_{p}}-\boldsymbol{x}_{s_{p}}\right)^{\top}\left(\boldsymbol{x}_{v_{p}}-\boldsymbol{x}_{s_{p}}\right)}+v_{k}
$$

where $v_{k} \sim \mathcal{N}\left(0, R_{k}\right)$. The measurement covariance $R_{k}$, shown here in units of distance, represents the imprecision in timing multiplied by the speed of sound. We can rewrite (35) in matrix notation as

$$
z_{k}=\left(\boldsymbol{x}_{p}^{\top} \boldsymbol{M}^{\top} \boldsymbol{M} \boldsymbol{x}_{p}\right)^{\frac{1}{2}}+v_{k}
$$

where $\boldsymbol{M}=\left[\begin{array}{ll}\boldsymbol{I} & -\boldsymbol{I}\end{array}\right]$. 
The Jacobian of the measurement, $\boldsymbol{H}_{k}$, with respect to $\boldsymbol{x}_{p}$ is then

$$
\begin{aligned}
\boldsymbol{H}_{k} & =\left.\frac{\partial z\left(\boldsymbol{x}_{p}\right)}{\partial \boldsymbol{x}_{p}}\right|_{\boldsymbol{x}_{p}=\hat{\boldsymbol{x}}_{p_{k \mid k-1}}} \\
& =\left(\hat{\boldsymbol{x}}_{p_{k \mid k-1}}^{\top} \boldsymbol{M}^{\top} \boldsymbol{M} \hat{\boldsymbol{x}}_{p_{k \mid k-1}}\right)^{-\frac{1}{2}} \hat{\boldsymbol{x}}_{p_{k \mid k-1}}^{\top} \boldsymbol{M}^{\top} \boldsymbol{M} .
\end{aligned}
$$

Substituting the measurement Jacobian into (5) and (8), the measurement update equation for this implementation of the extended Kalman filter becomes

$$
\begin{aligned}
\hat{\boldsymbol{x}}_{p_{k \mid k}} & =\hat{\boldsymbol{x}}_{p_{k \mid k-1}}+\boldsymbol{K}_{k}\left(\boldsymbol{z}_{k}-\left(\hat{\boldsymbol{x}}_{p_{k \mid k-1}}^{\top} \boldsymbol{M}^{\top} \boldsymbol{M} \hat{\boldsymbol{x}}_{p_{k \mid k-1}}\right)^{\frac{1}{2}}\right) \\
\boldsymbol{K}_{k} & =\boldsymbol{P}_{k \mid k-1} \boldsymbol{H}_{k}^{\top}\left(\boldsymbol{H}_{k} \boldsymbol{P}_{k \mid k-1} \boldsymbol{H}_{k}^{\top}+R_{k}\right)^{-1}
\end{aligned}
$$

Measurements from additional navigation sensors, e.g. depth sensor, gyrocompass, and Doppler velocity log, are processed asynchronously using standard observation models.

\section{G. Initialization}

Because the EKF algorithm performs linearization along the system trajectories, an initial state estimate too far from the actual state could cause the estimate to be unstable. In our implementation we initialize the EKF with a maximumlikelihood estimate (MLE) of the vehicle state and covariance. For this implementation of the EKF, the maximumlikelihood estimation is performed over the entire data set as previously described in [14]. For implementation in realtime, a maximum-likelihood estimate would be calculated over the first few range measurements and used to initialize the EKF.

\section{Field Results}

Sea trials were conducted during a cruise on the R/V Knorr to the southern Mid-Atlantic Ridge in January 2008. The goal of the cruise was to test and evaluate engineering methods for locating and mapping new hydrothermal vents on the southern Mid-Atlantic Ridge (SMAR).

\section{A. Site Description}

The southern Mid-Atlantic Ridge is formed by a divergent boundary between the South American Plate and the African Plate that is presently spreading at about $2.5 \mathrm{~cm}$ per year [21]. Our operations were conducted on a section of the SMAR to the north of the sites where active hydrothermal vents were first discovered by a combination of deep-tow and deep-submergence technologies culminating in photography by ABE [21] and subsequently sampled by the ROV Marum Quest [22]. The survey site, shown in Figure 2, is located near $04^{\circ} \mathrm{S} 12^{\circ} \mathrm{W}$ in a deep non-transform discontinuity whose maximum depth exceeds $4000 \mathrm{~m}$ [21].

\section{B. Experimental Setup}

The data presented here was collected by the autonomous underwater vehicle (AUV) Puma, developed at Woods Hole Oceanographic Institution [45]. Puma is a $5000 \mathrm{~m}$ rated AUV equipped with the following navigation sensors: a Paroscientific pressure depth sensor, an OCTANS fiber-optic gyrocompass for attitude and attitude rate measurements, and a $300 \mathrm{kHz}$ RDI Doppler velocity log (DVL) for velocity measurements. The vehicle is also equipped with a WHOI acoustic modem [15] and ITC-3013 transducer, which provide low-bandwidth data communication and two-way acoustic ranging. For these experiments the modem was equipped with a precision clock, developed by the authors [13], [14], which enables the micro-modem to measure oneway travel times for all received acoustic data packets.

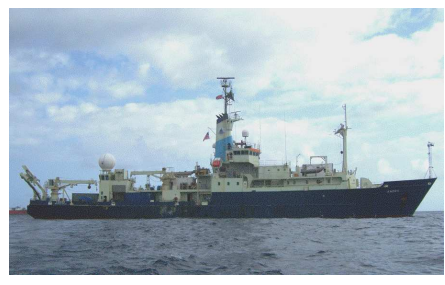

(a)

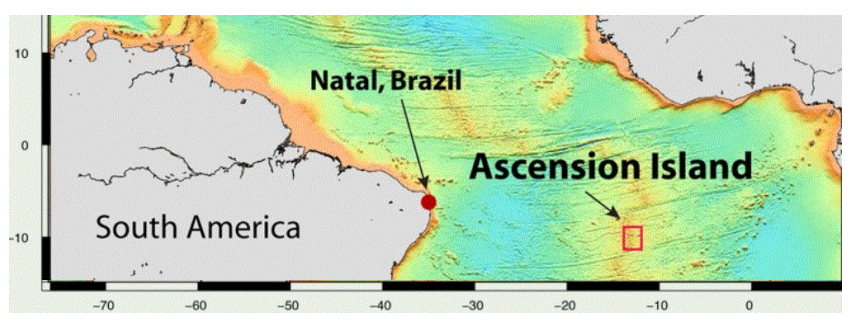

(c)

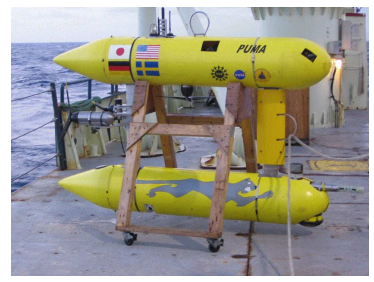

(b)
Fig. 2. (a) R/V Knorr (b) AUV Puma (c) The survey site is shown by the red box southeast of Ascension island on the southern Mid-Atlantic Ridge.

The R/V Knorr is a $279 \mathrm{ft}$ oceanographic research vessel operated by WHOI. The ship has two azimuthing stern thrusters, a retractable azimuthing bow thruster and dynamic positioning (DP) capability enabling it to hold station and maneuver in any direction [24]. For the ship's position information we used the C-Nav 2000 Real-Time GIPSY (RTG) GPS with a reported horizontal accuracy of $10 \mathrm{~cm}$ [10]. An Applanix POS/MV-320 provided heading, pitch, and roll data with a reported accuracy of $0.02^{\circ}$ [2]. The ship is also equipped with a WHOI micro-modem [15] and an ITC-3013 transducer for sending and receiving acoustic data packets. Figure 2 shows the R/V Knorr, the AUV Puma and the survey area in the red box southeast of Ascension Island.

The vehicle was programmed to conduct a survey comprised of 12 tracklines approximately 65 meters apart and 700 meters long while maintaining an altitude of $200 \mathrm{~m}$. While the vehicle was surveying, we repositioned the R/V Knorr around the survey site in a diamond shaped pattern, holding station at each apex. This was done to provide range fixes to the vehicle from different locations for increased observability. During these field trials the vehicle initiated all acoustic traffic. This conservative approach ensured that broadcasts of the vehicle's state and health would not be 


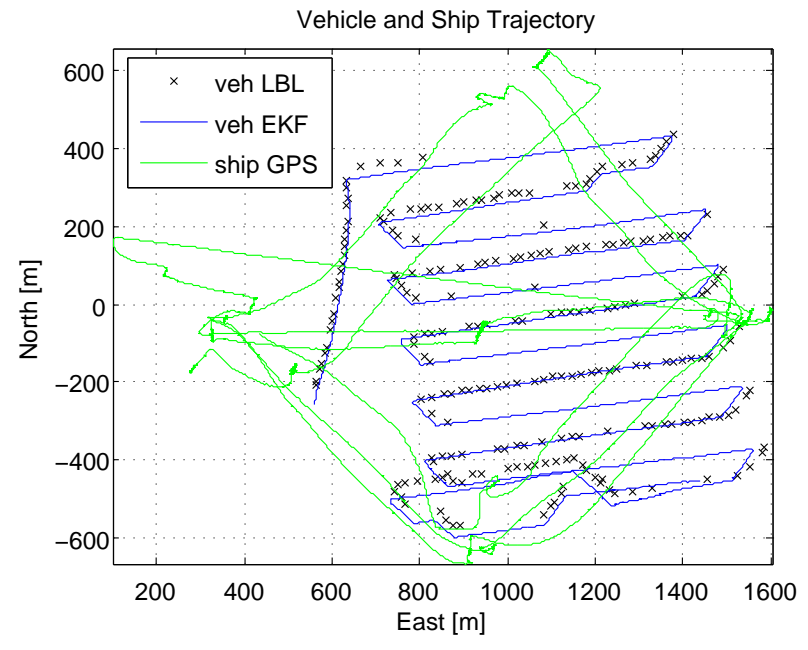

Fig. 3. EKF estimate of vehicle position compared to LBL fixes with an overlay of the ship's track.
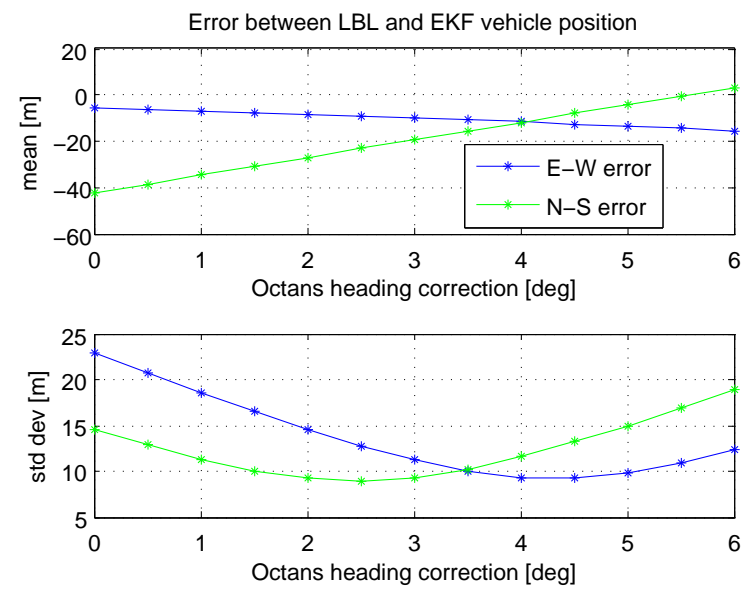

Fig. 4. East-West and North-South components of the error between the EKF-estimated vehicle position and the LBL vehicle position. Errors are calculated over the entire trackline for a range of OCTANS heading offsets.

reliant on the vehicle first successfully receiving a data request from the ship. Acoustic data packets were sent from the vehicle to the ship and requested by the vehicle from the ship every 30 seconds.

\section{Results}

The integrity of the acoustic channel varied over the course of the dive. While the vehicle was surveying near the bottom, on average one acoustic data packet from which we could calculate range was successfully received every 90 seconds.

Figure 3 shows an $\mathrm{XY}$ plot of the vehicle trajectory as estimated by the EKF compared with the vehicle position fixes from LBL. LBL fixes were largely unavailable on tracklines where the vehicle is moving to the East, most likely due to shadowing of the transducer by the vehicle frame at this vehicle heading.

Doppler attitude measurements were not used by the EKF, but we used them in post-processing in comparison to the OCTANS pitch and roll measurements to calibrate the offset between these two sensors. The means of the differences in pitch and roll measurements, $-3.24^{\circ}$ and $0.64^{\circ}$ respectively, are accounted for as mounting offsets in the OCTANS. The OCTANS heading offset was estimated by analyzing the mean and standard deviation of the error between the EKFestimated vehicle position and the LBL vehicle position over the entire trackline for various OCTANS heading offsets, shown in Figure 4, assuming the previously stated roll and pitch offsets. Given these data, a $3.5^{\circ}$ heading offset in the gyrocompass was assumed, resulting in a standard deviation of $10.2 \mathrm{~m}$ in both the East-West and the NorthSouth directions and means between $-10.3 \mathrm{~m}$ and $-13.5 \mathrm{~m}$.

These errors compare favorably with LBL, which has 1$10 \mathrm{~m}$ typical accuracy. However, the non-zero mean indicates the presence of a systematic errors that are not accounted for in the reported sensor calibrations. The authors are currently pursuing a more rigorous evaluation of sensor calibration.

\section{CONCLUSIONS}

This paper reports the first results from deep-water sea trials evaluating a synchronous-clock one-way-travel-time navigation method and comparing the results to an independent navigation source. An extended Kalman filter framework is described for combining vehicle navigation sensor data, ship navigation sensor data, and one-travel-time data between the vehicle and the ship for estimating the vehicle's trajectory in post-processing. Future research in this area will focus on the implementation of this navigation method in real-time and the development of a decentralized, recursive estimation framework to support multi-vehicle navigation with intervehicle ranging.

\section{ACKNOWLEDGMENTS}

This work was supported by the National Science Foundation under NSF Awards ATM-0427220 and ATM-0428122. The authors are grateful to Clay Kuntz and Chris Murphy for their support of Puma software, to Dr. Michael C. Jakuba for his support of the reported LBL navigation, and to Dr. James C. Kinsey for his help with sensor calibrations. The authors are also grateful to Captain George Silva, the Officers, and Crew of the R/V Knorr for their exemplary support.

\section{REFERENCES}

[1] J. C. Alleyne, "Position estimation from range only measurements," Master's thesis, Naval Postgraduate School, Monterey, California, 2000

[2] POS MV (Position and Orientation Systems for Marine Vessels), Applanix, Richmond Hill, ON, Canada, Nov. 2008, http://www.applanix.com/media/downloads/products/specs/ posmv_datasheet.pdf.

[3] P. Baccou and B. Jouvencel, "Homing and navigation using one transponder for AUV, postprocessing comparisons results with long base-line navigation," in Proc. IEEE Intl. Conf. Robot. Auto., vol. 4 , 2002, pp. 4004-4009.

[4] — "Simulation results, post-processing experimentations and comparisons results for navigation, homing and multiple vehicles operations with a new positioning method using on transponder," in Proc. IEEE/RSJ Intl. Conf. Intell. Robots Systems, vol. 1, Oct. 2003, pp $811-817$.

[5] A. Bahr, "Cooperative localization for autonomous underwater vehicles," Ph.D. dissertation, Massachusetts Institute of Technology, Cambridge, MA, USA, February 2009. 
[6] A. Bahr and J. Leonard, "Cooperative localization for autonomous underwater vehicles," in Proc. 10th International Symposium on Experimental Robotics (ISER), Rio de Janeiro, Brasil, july 2006.

[7] Y. Bar-Shalom, X. Rong Li, and T. Kirubarajan, Estimation with applications to tracking and navigation. New York: John Wiley \& Sons, Inc., 2001.

[8] J.-L. Blanco, J.-A. Fernandez-Madrigal, and J. Gonzalez, "Efficient probabilistic range-only slam," Intelligent Robots and Systems, 2008. IROS 2008. IEEE/RSJ International Conference on, pp. 1017-1022, Sept. 2008.

[9] J.-L. Blanco, J. Gonzalez, and J.-A. Fernandez-Madrigal, "A pure probabilistic approach to range-only slam," Robotics and Automation, 2008. ICRA 2008. IEEE International Conference on, pp. 1436-1441, May 2008.

[10] C-Nav2000, C\&C Technologies, Lafayette, LA, USA, http://www.cctechnol.com/printable.php?pageID=77.

[11] D. Djugash, S. Singh, and P. Corke, Field and Service Robotics, ser. Springer Tracts in Advanced Robotics. Berlin, Heidelberg: Springer Berlin / Heidelberg, 2006, vol. 25, ch. Further Results with Localization and Mapping Using Range from Radio, pp. 231-242.

[12] J. Djugash, S. Singh, G. Kantor, and W. Zhang, "Range-only slam for robots operating cooperatively with sensor networks," Robotics and Automation, 2006. ICRA 2006. Proceedings 2006 IEEE International Conference on, pp. 2078-2084, May 2006.

[13] R. M. Eustice, L. L. Whitcomb, H. Singh, and M. Grund, "Recent advances in synchronous-clock one-way-travel-time acoustic navigation," in Proc. IEEE/MTS OCEANS Conf. Exhib., Boston, MA, USA, Sep. 2006, pp. 1-6.

[14] — , "Experimental results in synchronous-clock one-way-travel-time acoustic navigation for autonomous underwater vehicles," in Proc. IEEE Intl. Conf. Robot. Auto., Rome, Italy, Apr. 2007, pp. 4257-4264.

[15] L. Freitag, M. Grund, S. Singh, J. Partan, P. Koski, and K. Ball, "The WHOI micro-modem: an acoustic communications and navigation system for multiple platforms," in Proc. IEEE/MTS OCEANS Conf. Exhib., Washington, D.C., Sep. 2005, pp. 1086-1092.

[16] A. Gadre, "Observability analysis in navigation systems with an underwater vehicle application," Ph.D. dissertation, Virginia Polytechnic Institute and State University, Blacksburg, Virginia, Jan. 2007.

[17] A. Gadre and D. Stilwell, "Toward underwater navigation based on range measurements from a single location," in Proc. IEEE Intl. Conf. Robot. Auto., vol. 5, 2004, pp. 4472-4477.

[18] _ "A complete solution to underwater navigation in the presence of unknown currents based on range measurements from a single location," in Proc. IEEE/RSJ Intl. Conf. Intell. Robots Systems, 2005, pp. 1420-1425.

[19] —, "Underwater navigation in the presence of unknown currents based on range measurements from a single location," American Control Conference, 2005. Proceedings of the 2005, vol. 1, pp. 656661, Jun. 2005.

[20] A. Gelb, Ed., Applied optimal estimation. Cambridge, MA: MIT Press, 1982

[21] C. German, S. Bennett, D. Connelly, A. Evans, B. Murton, L. Parson, R. Prien, E. Ramirez-Llodra, M. Jakuba, T. Shank, D. Yoerger, E. Baker, S. Walker, and K. Nakamura, "Hydrothermal activity on the southern mid-atlantic ridge: Tectonically- and volcanically-controlled venting at 4-5s," Earth and Planetary Science Letters, vol. 273, no. 3-4, pp. 332 - 344, 2008. [Online]. Available: http://www.sciencedirect.com/science/article/B6V61-4SXP6TF1/2/976b220c0df55ef271a38c9c24f2c5a8

[22] K. M. Haase, S. Petersen, A. Koschinsky, R. Seifert, C. W. Devey, R. Keir, K. S. Lackschewitz, B. Melchert, M. Perner, O. Schmale, J. Süling, N. Dubilier, F. Zielinski, S. Fretzdorff, D. Garbe-Schönberg, U. Westernströer, C. R. German, T. M. Shank, D. Yoerger, O. Giere, J. Kuever, H. Marbler, J. Mawick, C. Mertens, U. Stöber, M. Walter, C. Ostertag-Henning, H. Paulick, M. Peters, H. Strauss, S. Sander, J. Stecher, M. Warmuth, and S. Weber, "Young volcanism and related hydrothermal activity at $5 \mathrm{degS}$ on the slow-spreading southern MidAtlantic Ridge," Geochemistry, Geophysics, Geosystems, vol. 8, pp. $11002-+$, Nov. 2007.

[23] J. C. Hartsfield, "Single transponder range only navigation geometry (STRONG) applied to REMUS autonomous under water vehicles," Master's thesis, Massachusetts Institute of Technology and Woods Hole Oceanographic Institution, Aug. 2005.

[24] W. H. O. Institution, "R/V Knorr: Specifications," http://www.whoi.edu/page.do?pid=8496, Woods Hole, MA.
[25] J. Jouffroy and J. Reger, "An algebraic perspective to singletransponder underwater navigation," in Proceedings IEEE 2006 CCA/CACSD/ISIC, Munich, Germany, 2006.

[26] R. E. Kalman, "A new approach to linear filtering and prediction problems," Transactions of the ASME-Journal of Basic Engineering, vol. 82, no. Series D, pp. 35-45, 1960.

[27] G. Kantor and S. Singh, "Preliminary results in range-only localization and mapping," in Proceedings of the 2002 IEEE International Conference on Robotics and Automation, 2002, pp. 1818-1823.

[28] A. Kehagias, J. Djugash, and S. Singh, "Range-only slam with interpolated range data," Robotics Institute, Pittsburgh, PA, Tech. Rep. CMU-RI-TR-06-26, May 2006.

[29] D. Kurth, G. Kantor, and S. Singh, "Experimental results in rangeonly localization with radio," Intelligent Robots and Systems, 2003. (IROS 2003). Proceedings. 2003 IEEE/RSJ International Conference on, vol. 1, pp. 974-979 vol.1, Oct. 2003.

[30] D. Kurth, "Range-only robot localization and slam with radio," Master's thesis, Carnegie Mellon University, Pittsburgh, PA, May 2004.

[31] C. E. LaPointe, "Virtual long baseline (vlbl) autonomous underwater vehicle navigation using a single transponder," Master's thesis, Massachusetts Institute of Technology and Woods Hole Oceanographic Institution, Jun. 2006.

[32] M. Laresen, "High performance autonomous underwater navigation: Experimental results," Hydro Intl., vol. 6, no. 1, pp. 6-9, Jan./Feb. 2002.

[33] M. Larsen, "Synthetic long baseline navigation of underwater vehicles," in Proc. IEEE/MTS OCEANS Conf. Exhib., vol. 3, Sep. 2000 , pp. 2043-2050

[34] M. B. Larsen, "Autonomous navigation of underwater vehicles," $\mathrm{Ph} . \mathrm{D}$ dissertation, Technical University of Denmark, Denmark, Feb. 2001.

[35] E. Larsson, "Cramer-rao bound analysis of distributed positioning in sensor networks," Signal Processing Letters, IEEE, vol. 11, no. 3, pp. 334-337, March 2004

[36] P.-M. Lee, B.-H. Jun, and Y.-K. Lim, "Review on underwater navigation system based on range measurements from one reference," OCEANS 2008 - MTS/IEEE Kobe Techno-Ocean, pp. 1-5, April 2008

[37] J. Marçal, J. Jouffroy, and T. I. Fossen, "An extended set-valued observer for position estimation using single range measurements," in Proceedings International Symposium on Unmanned Untethered Submersible Technology, Aug. 2005.

[38] E. Martinson and F. Dellaert, "Marco polo localization," Robotics and Automation, 2003. Proceedings. ICRA '03. IEEE International Conference on, vol. 2, pp. 1960-1965 vol.2, Sept. 2003.

[39] P. M. Newman and J. J. Leonard, "Pure range-only sub-sea slam," in Proceedings of the 2003 IEEE International Conference on Robotics and Automation, 2003, pp. 1921-1926.

[40] E. Olson, J. Leonard, and S. Teller, "Robust range-only beacon localization," IEEE Journal of Oceanic Engineering, pp. 949-958, Oct. 2006

[41] B. Ristic, S. Arulampalam, and J. McCarthy, "Target motion analysis using range-only measurements: algorithms, performance and application to ISAR data," Signal Processing, vol. 82, no. 2, pp. 273-296, Feb. 2002.

[42] A. Ross and J. Jouffroy, "Remarks on the observability of single beacon underwater navigation," in Proc. Intl. Symp. Unmanned Unteth. Subm. Tech., Aug. 2005.

[43] A. Scherbatyuk, "The AUV positioning using ranges from one transponder LBL," in Proc. IEEE/MTS OCEANS Conf. Exhib., vol. 3, San Diego, CA, 1995, pp. 1620-1623.

[44] H. Singh, J. Bellingham, F. Hover, S. Lerner, B. Moran, K. von der Heydt, and D. Yoerger, "Docking for an autonomous ocean sampling network," IEEE J. Oceanic Eng., vol. 26, no. 4, pp. 498-514, Oct. 2001

[45] H. Singh, A. Can, R. M. Eustice, S. Lerner, N. McPhee, O. Pizarro, and C. Roman, "SeaBED AUV offers new platform for high-resolution imaging," EOS, Trans. Amer. Geophysical Union, vol. 85, no. 31, pp. 289,294-295, Aug. 2004.

[46] T. Song, "Observability of target tracking with range-only measurements," IEEE Journal of Oceanic Engineering, vol. 24, no. 24, pp. 383-387, Jul. 1999.

[47] J. R. Spletzer, "A new approach to range-only slam for wireless sensor networks," Lehigh University, Bethlehem, PA, Tech. Rep., 2003.

[48] J. Vaganay, P. Baccou, and B. Jouvencel, "Homing by acoustic ranging to a single beacon," in Proc. IEEE/MTS OCEANS Conf. Exhib., vol. 2 , Sep. 2000, pp. 1457-1462. 